\title{
A Compact Prime Listing
}

\author{
By Sol Weintraub
}

\begin{abstract}
A very compact listing of the primes to 100000 is given and applications to computer storage of primes is indicated.
\end{abstract}

The accompanying table gives a compact listing of prime numbers for the interval from zero to 100000 . Each decade (an interval of 10 numbers) can contain at most 4 primes: Those numbers congruent to $1,3,7$ or $9 \bmod 10$ (with the obvious exception of the first four primes: 2, 3, 5 and 7). There exist $\sum_{i=0}^{4}\left(\begin{array}{l}4 \\ i\end{array}\right)=16$ possible combinations for each decade. These 16 possibilities are represented in the table by the symbols $\mathrm{O}, \mathrm{A}, \mathrm{B}, \mathrm{C}, \cdots, \mathrm{N}$ and $P$ and their meanings are indicated at the bottom of the table. (An additional symbol, $Q$, is used for the first four primes.)

The left-hand column in the table indicates the thousands, the top row the hundreds and the second row the decades. Thus, for example, to see if 39199 is prime we look at the row for 39000 in the 100 column and down the 9 column and find the symbol G. This shows that in this decade 1 and 9 are primes; i.e., 39191 and 39199 are both primes.

In addition, the table lists the counts of primes for each interval of 100 and for each 1000 . The key for the hundreds is indicated at the bottom of the first page of the table. Thus, there are $\mathrm{P}=25$ primes from 0 to 100 and $\mathrm{L}=21$ primes from 100 to 200 etc. From 0 to 1000 there are 168 primes. Also, as shown on the bottom of the table, there are 5133 primes from zero to 50000 and 9592 primes from zero to 100000 .

Since, in most computers, words are represented in the octal or hexadecimal (16) systems the listing provides an efficient means for computer storage of primes.

Queens College, CUNY

Flushing, New York 11367

Received March 14, 1973.

AMS (MOS) subject classifications (1970). Primary 10A25.

Key words and phrases. Primes, prime counts, computer storage.

Copyright (C) 1974. American Mathematical Society 

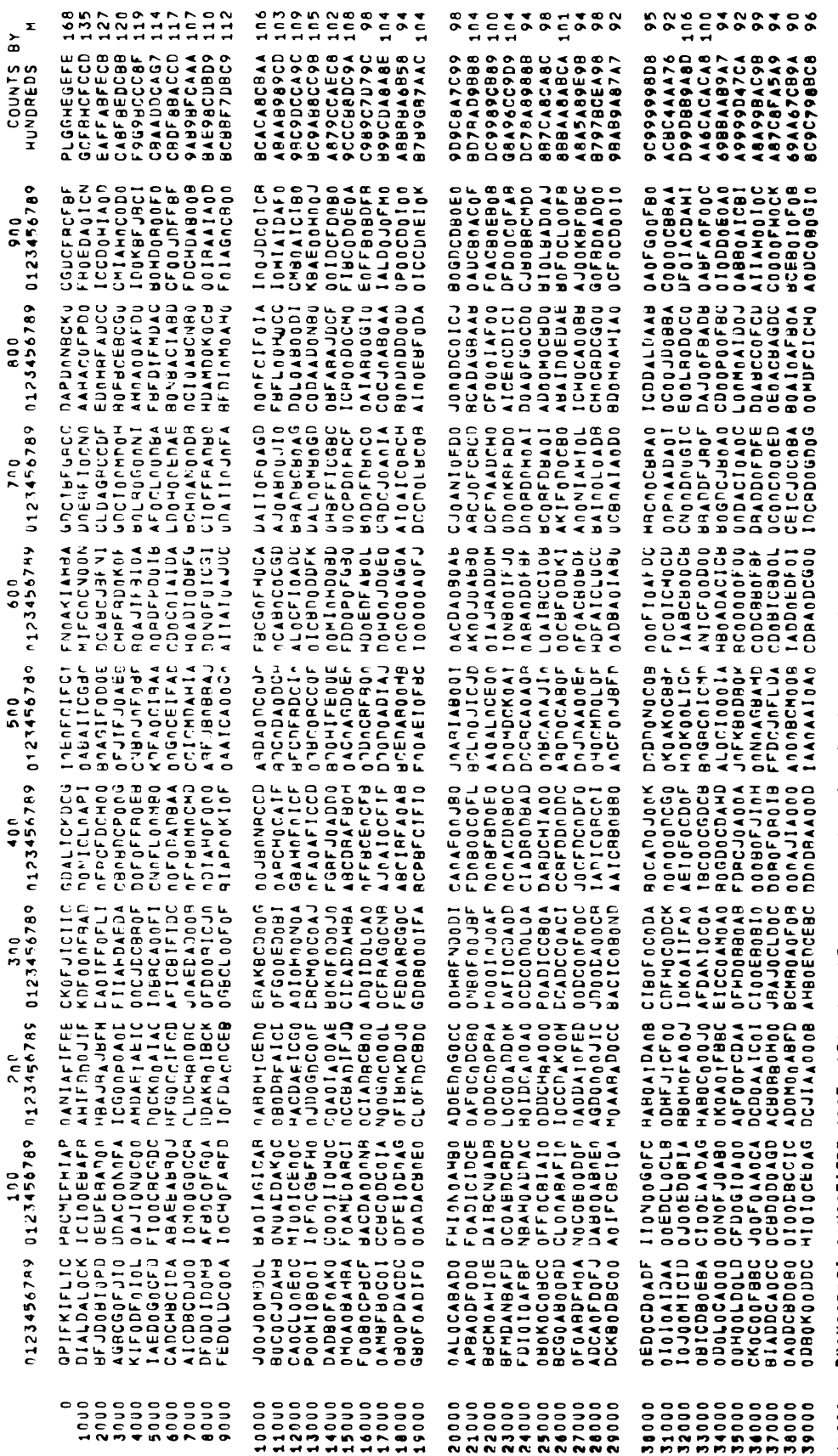

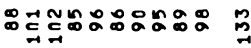

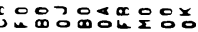

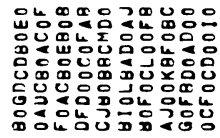

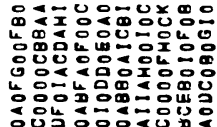

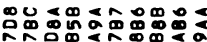

in

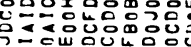

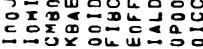

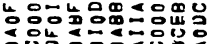

र́⿴囗十

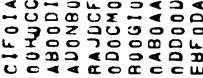

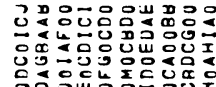

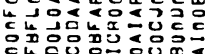

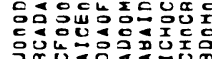

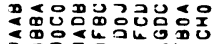

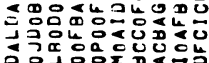

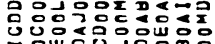

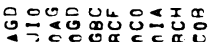

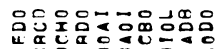

ix 要贾

우

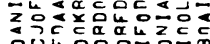

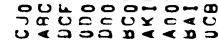

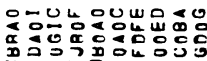

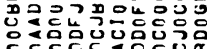

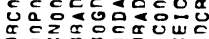

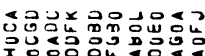

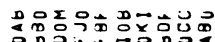

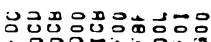

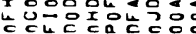

की

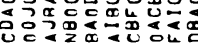

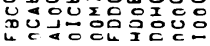

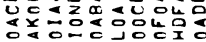

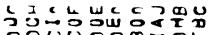

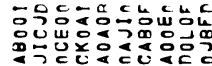

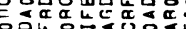

ब

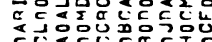

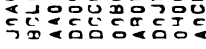

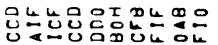

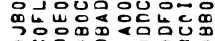

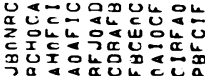

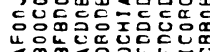

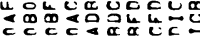

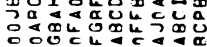

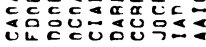

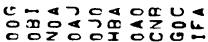

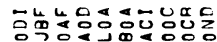

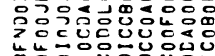

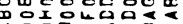

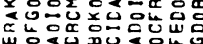

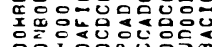

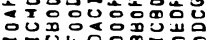

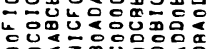

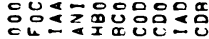

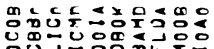

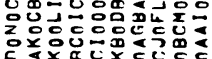

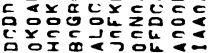

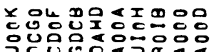

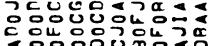

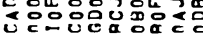

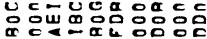

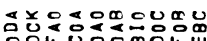

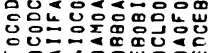

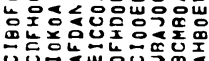

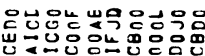

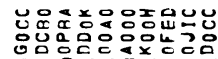

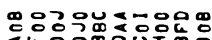

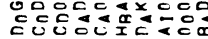

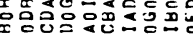

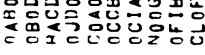

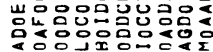

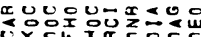

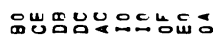

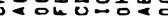

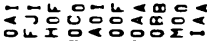

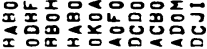

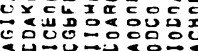

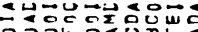

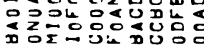

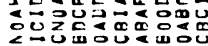

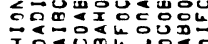

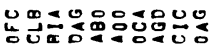

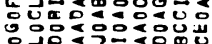

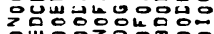

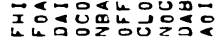

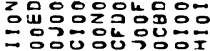

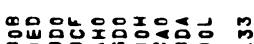

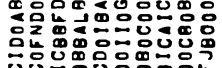

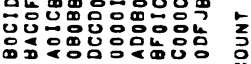

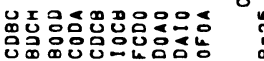

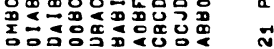

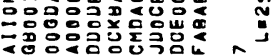

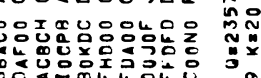

00.0

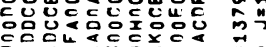

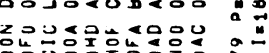

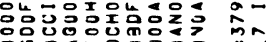

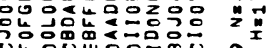

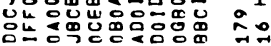

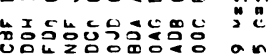

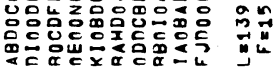

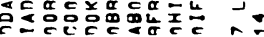

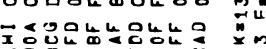

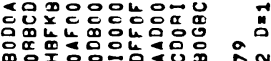

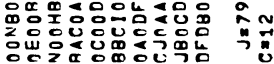

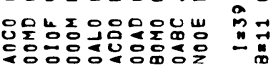

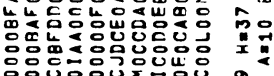

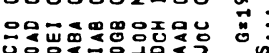

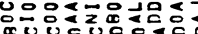

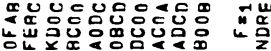



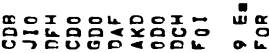

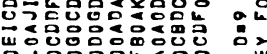

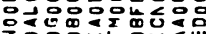

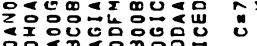

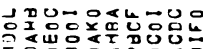

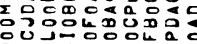

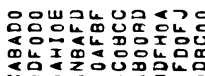

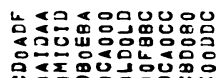

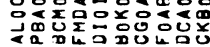

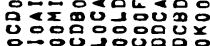

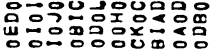

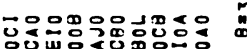

这

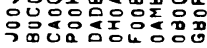

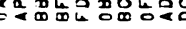

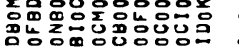

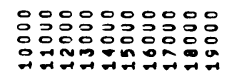

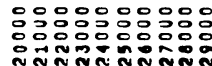

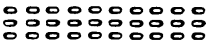

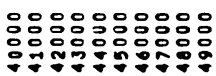




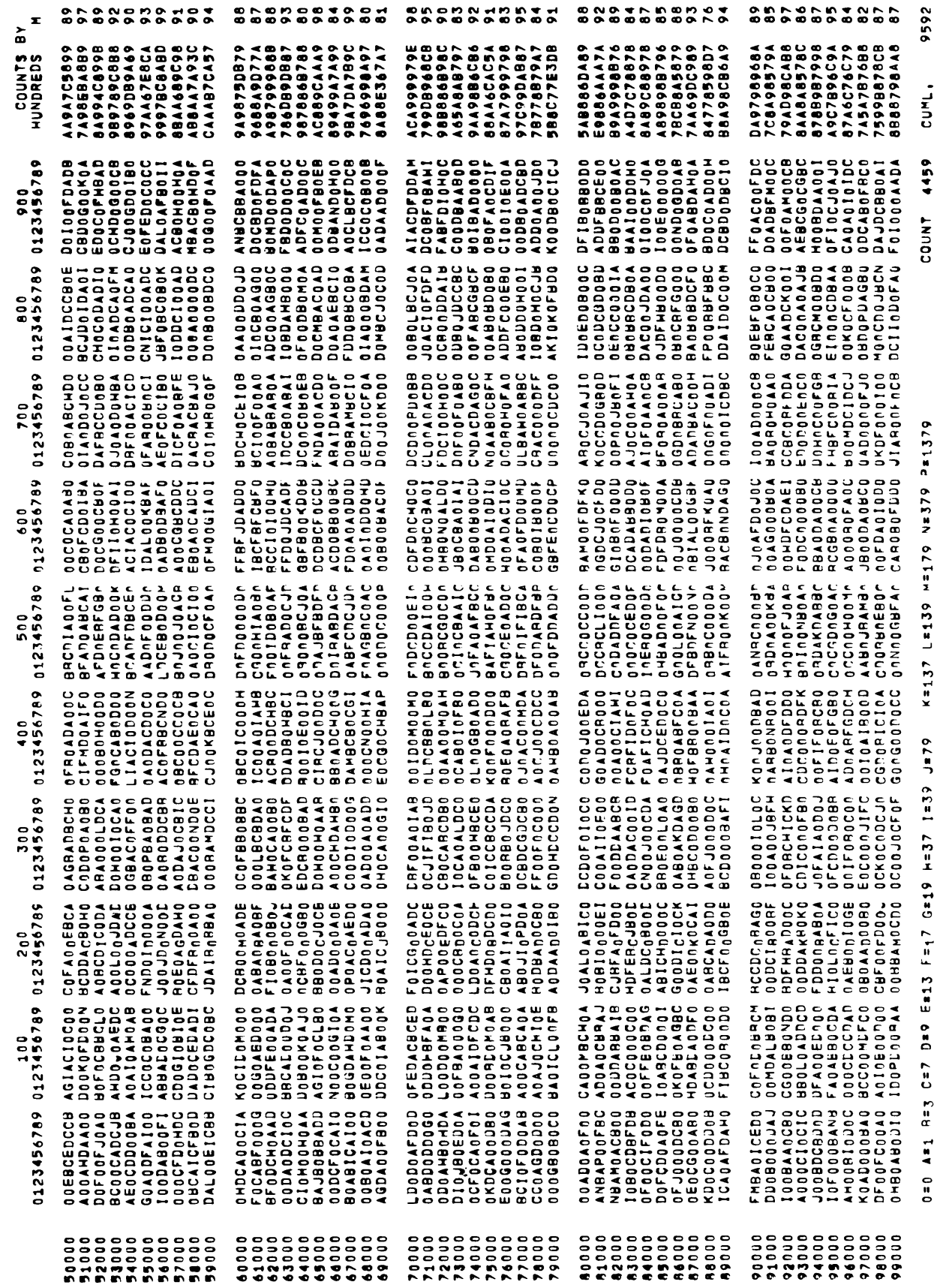

